\title{
Frequency gradients of DHCR7 mutations in patients with Smith-Lemli-Opitz syndrome in Europe: evidence for different origins of common mutations
}

\author{
M Witsch-Baumgartner ${ }^{1}$, E Ciara $^{2}$, J Löffler ${ }^{1}$, HJ Menzel $^{1}$, U Seedorf $^{3}$, J Burn ${ }^{4}$, \\ G Gillessen-Kaesbach ${ }^{5}$, GF Hoffmann ${ }^{6}$, BU Fitzky ${ }^{7}, \mathrm{H}_{\text {Mundy }}{ }^{8}$, P Clayton $^{8}$, RI Kelley ${ }^{9}$, \\ M Krajewska-Walasek ${ }^{2}$ and G Utermann ${ }^{1}$ \\ ${ }^{1}$ Institute of Medical Biology and Human Genetics, Innsbruck, Austria; ${ }^{2}$ Department of Medical Genetics, The \\ Children's M emorial Health Institute, Warsaw, Poland; ${ }^{3}$ Institute for Arteriosclerosis Research, M ünster, Germany; \\ ${ }^{4}$ University of Newcastle upon Tyne, School of Biochemistry and Genetics, Newcastle upon Tyne, UK; ${ }^{5}$ Institut für \\ Humangenetik, Universitätsklinikum Essen, Germany; ${ }^{6}$ Department of Neuropediatrics and M etabolic Disease, \\ University of Marburg, Germany; ${ }^{7}$ Institute of Biochemical Pharmacology, Innsbruck, Austria; ${ }^{8}$ Institute of Child \\ Health and Great Ormond Street Hospital, London, UK; ${ }^{9}$ Kennedy Krieger Institute and Department of Pediatrics, \\ Johns Hopkins University School of Medicine, Baltimore, ML, USA
}

Smith-Lemli-Opitz syndrome/ RSH (SLOS) is a multiple congenital anomaly syndrome caused by mutations in the gene for $\Delta^{7}$-sterol reductase (DHCR7) which catalyses the last step in the biosynthesis of cholesterol. SLOS is among the common recessive disorders in Europeans but almost absent in most other populations. More than $\mathbf{4 0}$ mutations in the DHCR7 gene some of which are frequent have been described in SLOS patients of various origins. Here we report mutation analysis of the DHCR7 gene in SLOS patients from Poland $(n=15)$, Germany/Austria $(n=22)$ and Great Britain $(n=22)$. Altogether 35 different mutations were identified and the two null mutations IVS8-1G >C and W151X were the most frequent in the total sample. In all three populations three mutations accounted for $>0.5$ of SLOS chromosomes. The mutational spectra were, however, significantly different across these populations with each of the common mutations showing an east-west gradient (W151X, V326L) or vice versa (IVS8-1G > C). W151X is the most frequent (0.33) mutation in Polish SLOS patients. It has an intermediate frequency in German/Austrian patients (0.18) and is rare among British patients (0.02). V326L shows the same distribution pattern (Poland 0.23, Germany/Austria 0.18, Britain 0.02). In contrast IVS8-1G $>C$ is most frequent in Britain (0.34) intermediate in Germany/Austria (0.20) and rare in Poland (0.03). All analysed IVS8-1G > C and V326L alleles shared the same DHCR7 haplotype, whereas the W151X mutation occurred on different haplotypes. There is evidence for both recurrent mutations and founder effects. Together this suggests that the common SLOS mutations in Europe have different geographic and historic origins and spread across the continent in opposite directions. European Journal of Human Genetics (2001) 9, 45-50.

\section{Keywords: SLOS; mutational spectra; cholesterol; population genetics}

\section{Introduction}

The Smith-Lemli-Opitz/RSH syndrome (SLOS) [MIM $270400]^{1}$ is an autosomal recessive disorder with an estimated frequency of $1: 15.000$ to $1: 40.000$ in European

Correspondence: Professor Dr Gerd Utermann, Institute of Medical Biology and Human Genetics, Schoepfstrasse 41, A-6020 Innsbruck, Austria. Tel: +43512 507 3450; Fax: +43512507 2861; E-mail: gerd.utermann@uibk.ac.at

Received 17 May 2000; revised 21 August 2000; accepted 13 September 2000 populations. $^{2-4}$ The syndrome is reportedly common in the Czech Republic and Slovakia and is rare or absent in nonCaucasian populations. SLOS is the prototype of a severe human malformation syndrome which is caused by an inborn error of metabolism. ${ }^{5-7}$ The syndrome is characterised by a variable combination of malformations, dysmorphic signs and mental retardation including growth retardation, microcephaly, genital anomalies in males, postaxial polydactyly and 2/3syndactyly of toes. Severity ranges from intrauterine lethality to mild dysmorphism and moderate 
mental impairment. ${ }^{2,8,9}$ Diagnosis may be difficult at both ends of the clinical spectrum. ${ }^{10,11}$

A breakthrough in the knowledge of the etiology and pathogenesis of the SLOS was the demonstration by Irons, Tint and colleagues that SLOS is caused by an error in the biosynthesis of cholesterol. ${ }^{12,13}$ The last step in the biosynthesis of cholesterol is the conversion of 7-dehydrocholesterol $(7 \mathrm{DHC})$ to cholesterol which is catalysed by the enzyme $\Delta 7$-sterol reductase (DHCR7, EC13121) in the endoplasmatic reticulum. This step is defective in patients with $\mathrm{SLOS}^{13}$ resulting in low cholesterol and accumulation of the precursors 7DHC, 8DHC and others in plasma and cells. This allows for the biochemical diagnosis of the syndrome. ${ }^{9}$ Recently the gene coding for DHCR7 has been cloned ${ }^{14}$ and mutations in the gene were identified in all patients with biochemically confirmed SLOS. ${ }^{15-20}$ The mutational spectrum in the DHCR7 gene from a large group of patients with SLOS has been determined and a genotype-phenotype correlation has been established. ${ }^{18}$ Altogether over 40 DHCR7 mutations have been described in SLOS patients some of which were frequent. The three most common mutations identified in patients of European and EuropeanAmerican ancestry included two null mutations, ie IVS8IG >C and W151X, which in homozygotes or compounds are associated with the most severe phenotypes. ${ }^{18,21}$

In order to elucidate the origin and age of SLOS-causing mutations we have started to analyse DHCR7 gene mutations in SLOS patients from different European countries. Here we report the mutational spectra of SLOS mutations in three European populations and demonstrate significant differences in the frequency spectra of SLOS mutations across these populations. The frequency distributions and haplotype analysis suggest multiple origins of SLOS mutations in Europe.

\section{Patients and methods \\ Patients}

The study population included 59 unrelated patients with SLOS. They were recruited from various clinical genetic centres in Austria $(n=3)$, Germany $(n=19)$, Great Britain $(n=22)$ and Poland $(n=15)$. For the frequency analysis the Austrian and German patients were combined. All 59 patients had characteristic clinical features of SLOS and for 56 patients it was possible to confirm the clinical diagnosis by biochemical analysis of sterols (quantification by gas chromatography/mass spectrometry). ${ }^{22}$ A detailed clinical and biochemical characterisation of 12 of the Polish SLOS patients has been published. ${ }^{23}$ Eight of the British patients were recruited in the USA (Dr R Kelley). For these patients a British parental and grand-parental origin was known.

Sixteen of the Austrian/German and 12 of the British patients were from the large collection of SLOS patients reported previously. ${ }^{18}$ For haplotype analysis also patients from a previous study were considered. ${ }^{18}$

\section{Mutation and haplotype analysis}

Amplification of DNA, SSCP, DGGE and sequencing were performed exactly as described ${ }^{18}$ using the primers and experimental condition described therein. For construction of haplotypes eight single nucleotide polymorphisms in the coding sequence of the DHCR7 gene ${ }^{18}$ (M Witsch-Baumgartner and G Utermann, 2000, unpublished) were analysed by sequencing of the respective exons from patients homozygous for the respective mutations and from compound patients and their parents.

\section{Results}

Using a combination of SSCP, DGGE and direct sequencing ${ }^{18}$ we analysed all exons including exon/intron boundaries of the DHCR7 gene from 22British, 22German and Austrian and 15 Polish patients with SLOS for mutations. Altogether 35 different mutations were present in the total group. Twenty-six of these have been previously identified by us ${ }^{15,18}$ and others. ${ }^{16,17,19,24-26}$ Six of the mutations have not been described before (T154R, M270V, G309S, R443H, I178N, $\mathrm{R} 242 \mathrm{H}$ ). No mutation was found on four out of 118SLOS chromosomes from four patients $(96.6 \%$ mutation-detection rate). The spectrum of mutations was most heterogeneous in the British SLOS patients and more homogeneous in the Polish patients. The most frequent mutations in the total same were IVS8-1G >C (0.21) W151X (0.16), and V326L (0.14).

\section{Spectrum of DHCR7 mutations in Polish SLOS patients}

The mutational spectrum in thePolish patients was distinctly different from the ones in British and German/Austrians (Table1). Altogether 11 different mutations were identified. Among the Polish SLOS patients three mutations (W151X, V326L, R352W) were present on $>0.7$ of SLOS chromosomes. These mutations were also present in the other populations

Table 1 Spectrum of SLOS-causing mutations in the DHCR7 gene in 15 Polish patients

\begin{tabular}{|c|c|c|c|c|c|c|}
\hline Mutation & $\begin{array}{l}\text { Class of } \\
\text { mutation }\end{array}$ & Exon & $\begin{array}{l}\text { No. of } \\
\text { alleles } \\
(n=30)\end{array}$ & Frequency & Expected & Identified \\
\hline W151X & 0 & 6 & 10 & 0.333 & 1.66 & - \\
\hline V326L & TM & 9 & 7 & 0.233 & 0.81 & 1 \\
\hline R352W & TM & 9 & 4 & 0.133 & 0.27 & - \\
\hline L157P & TM & 6 & 2 & 0.067 & 0.067 & 1 \\
\hline IVS8-1G>C & 0 & 9 & 1 & 0.033 & 0.016 & - \\
\hline P51S & TM & 4 & 1 & 0.033 & 0.016 & - \\
\hline$M 270 V^{b}$ & TM & 7 & 1 & 0.033 & 0.016 & - \\
\hline G309S & TM & 8 & 1 & 0.033 & 0.016 & - \\
\hline R446Q & $\mathrm{CT}$ & 9 & 1 & 0.033 & 0.016 & - \\
\hline $\mathrm{R} 443 \mathrm{H}^{\mathrm{b}}$ & $\mathrm{CT}$ & 9 & 1 & 0.033 & 0.016 & - \\
\hline $1178 \mathrm{~N}^{\mathrm{b}}$ & TM & 6 & 1 & 0.033 & 0.016 & - \\
\hline
\end{tabular}

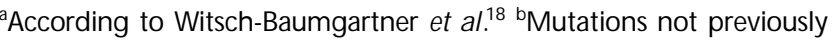
identified. TM =transmembrane, CT=C-terminal, $0=$ null mutation. 
though at lower frequencies. Five of the rare Polish mutations each represented by a single chromosome were not observed in the other two populations (R446Q, M270V, G309S, R443H, I178N).

\section{Spectrum of DHCR7 mutations in British SLOS patients}

Twenty-one different mutations and one unknown were present in the British SLOS patients (Table2). The splice site mutation IVS8-1G >C was the most frequent and accounted for one third (0.34) of British SLOS chromosomes. This mutation has also been identified as the most frequent in a mixed American/European sample of SLOS patients ${ }^{18}$ and in an American SLOS population. ${ }^{19}$ The relative frequency of the IVS8-1G >C mutation was even higher (0.46) when only SLOS patients born and identified in Great Britain were considered $(n=14)$. None of the other mutations had a frequency of $>0.1$. The mutation R404C had a frequency of 0.091 . The third most frequent mutation (0.068) in the British T93M was recently identified as the most frequent mutation among Italian SLOS patients. ${ }^{24}$ All other British SLOS mutations were rare and represented by only one or two alleles.

\section{Spectrum of DHCR7 mutations in German and Austrian SLOS patients}

Sixteen different mutations were identified on 44 chromosomes (Table3). In three of the biochemically analysed SLOS

Table 2 Spectrum of SLOS-causing mutations in the DHCR7 gene in 22 British patients

\begin{tabular}{|c|c|c|c|c|c|c|}
\hline Mutation & $\begin{array}{l}\text { Class of } \\
\text { mutation }^{a}\end{array}$ & Exon & $\begin{array}{l}\text { No. of } \\
\text { alleles } \\
(n=44)\end{array}$ & Frequency & No. of hor & mozygotes \\
\hline IVS8-1G >C & 0 & 9 & 15 & 0.34 & 2.5 & 2 \\
\hline R404C & $4 \mathrm{~L}$ & 9 & 4 & 0.091 & 0.18 & 1 \\
\hline T93М & TM & 4 & 3 & 0.068 & 0.1 & - \\
\hline R242C & TM & 7 & 2 & 0.045 & 0.04 & - \\
\hline E448K & $\mathrm{CT}$ & 9 & 2 & 0.045 & 0.04 & - \\
\hline F284L & TM & 8 & 2 & 0.045 & 0.04 & - \\
\hline E448Q & CT & 9 & 1 & 0.023 & 0.01 & - \\
\hline P51S & TM & 4 & 1 & 0.023 & 0.01 & - \\
\hline Q107H & TM & 4 & 1 & 0.023 & 0.01 & - \\
\hline del 385-412 & 0 & 5 & 1 & 0.023 & 0.01 & - \\
\hline W151X & 0 & 6 & 1 & 0.023 & 0.01 & - \\
\hline G147D & TM & 6 & 1 & 0.023 & 0.01 & - \\
\hline $\mathrm{T} 154 \mathrm{R}^{\mathrm{b}}$ & TM & 6 & 1 & 0.023 & 0.01 & - \\
\hline T289I & TM & 8 & 1 & 0.023 & 0.01 & - \\
\hline Y324H & TM & 9 & 1 & 0.023 & 0.01 & - \\
\hline V326L & TM & 9 & 1 & 0.023 & 0.01 & - \\
\hline Y408H & $4 \mathrm{~L}$ & 9 & 1 & 0.023 & 0.01 & - \\
\hline G410R & 4L & 9 & 1 & 0.023 & 0.01 & - \\
\hline G410S & 4L & 9 & 1 & 0.023 & 0.01 & - \\
\hline R450L & CT & 9 & 1 & 0.023 & 0.01 & - \\
\hline F302L & TM & 8 & 1 & 0.023 & 0.01 & - \\
\hline unknown & $?$ & $?$ & 1 & 0.023 & & \\
\hline
\end{tabular}

${ }^{a}$ According to Witsch-Baumgartner et al. ${ }^{18}$ bMutations not previously identified. $\mathrm{TM}=$ transmembrane, $\mathrm{CT}=\mathrm{C}$-terminal, $0=$ null mutation, 4L=4th cytoplasmic loop.
Table 3 Spectrum of SLOS-causing mutations in the DHCR7 gene in 22 German/Austrian patients

\begin{tabular}{|c|c|c|c|c|c|c|}
\hline Mutation & $\begin{array}{l}\text { Class of } \\
\text { mutation }{ }^{a}\end{array}$ & Exon & $\begin{array}{l}\text { No. of } \\
\text { alleles } \\
(n=44)\end{array}$ & Frequency & No. of hor & nozygotes \\
\hline IVS8-1G>C & 0 & 9 & 9 & 0.20 & 0.9 & 2 \\
\hline V326L & TM & 9 & 8 & 0.18 & 0.7 & - \\
\hline W151X & 0 & 6 & 8 & 0.18 & 0.7 & 1 \\
\hline R352W & TM & 9 & 3 & 0.07 & 0.10 & - \\
\hline L99P & TM & 4 & 2 & 0.05 & 0.06 & - \\
\hline P51S & TM & 4 & 1 & 0.02 & 0.01 & - \\
\hline H119L & TM & 5 & 1 & 0.02 & 0.01 & - \\
\hline L157P & TM & 6 & 1 & 0.02 & 0.01 & - \\
\hline R242C & TM & 7 & 1 & 0.02 & 0.01 & - \\
\hline $\mathrm{R} 242 \mathrm{H}^{\mathrm{b}}$ & TM & 7 & 1 & 0.02 & 0.01 & - \\
\hline A247V & TM & 7 & 1 & 0.02 & 0.01 & - \\
\hline R404C & $4 \mathrm{~L}$ & 9 & 1 & 0.02 & 0.01 & - \\
\hline C380S & $4 \mathrm{~L}$ & 9 & 1 & 0.02 & 0.01 & - \\
\hline G410S & 4L & 9 & 1 & 0.02 & 0.01 & - \\
\hline R443C & $\mathrm{CT}$ & 9 & 1 & 0.02 & 0.01 & - \\
\hline E448K & CT & 9 & 1 & 0.02 & 0.01 & - \\
\hline unknown & $?$ & $?$ & 3 & 0.07 & & \\
\hline
\end{tabular}

${ }^{a}$ According to Witsch-Baumgartner et al ${ }^{18}$ bMutation not previously identified. TM=transmembrane, $\mathrm{CT}=\mathrm{C}$-terminal, $\mathrm{O}=$ null mutation, $4 \mathrm{~L}=4$ th cytoplasmic loop.

patients no mutation could be identified on one chromosome. Three mutations (IVS8-1G > C, V326L and W151X) were frequent. For all three mutations frequencies were intermediate between the respective British and Polish frequencies.

\section{Haplotype analysis}

We used eight single nucleotide polymorphisms in the coding sequence of the DHCR7 gene to construct haplotypes: 189A > G; 207C > T; 231C > T; 438C > T, 969G >A, $1158 \mathrm{C}>\mathrm{T}, 1272 \mathrm{~T}>\mathrm{C}, 1350 \mathrm{C}>\mathrm{T}$ (Table4A).

In 24 patients haplotype analysis was performed and information was obtained for 47 mutant DHCR7 alleles. These represented 14 different mutations. Altogether we observed nine different haplotypes; 28 mutant DHCR7 chromosomes representing nine different mutations were all characterised by the same haplotype, whereas 19 mutant DHCR7 alleles (eight different mutations) occurred on eight different haplotypes (Table4B).

The six common mutations (IVS8-1G >C, R404C, T93M, W151X, V326L, R352W) occurred on seven different haplotypes. Three of the frequent mutations (R404C, T93M, $\mathrm{R} 352 \mathrm{~W}$ ) involve CpGs. Each of these mutations occurred on at least two different haplotypes which differed at more than one of the polymorphic sites (eg R352W occurred on haplotypes $A$ and I). Hence the high frequency of these mutations is most likely explained by recurrent mutations.

In contrast 13IVS8-1G >C and 5V326L chromosomes were all characterised by the same DHCR7 haplotype. Only one IVS8-1G >C mutation was present on a different haplotype. This mutation was present in cis with a T93M 


\section{Table 4}

A DHCR7 haplotypes in 24 SLOS patients

\begin{tabular}{llllllllll}
\hline $\begin{array}{l}\text { Base } \\
\text { position }\end{array}$ & \multicolumn{3}{c}{ Haplotypes } \\
& A & B & C & D & E & F & G & H & I \\
\hline 189 & A & G & A & A & G & G & G & G & G \\
207 & C & C & C & C & C & C & C & T & T \\
231 & C & C & C & T & T & C & C & C & T \\
438 & C & C & C & C & C & C & C & C & T \\
969 & G & G & G & G & G & G & G & G & G \\
1158 & C & C & C & C & C & C & C & T & T \\
1272 & T & T & C & T & T & C & C & C & C \\
1350 & C & C & C & C & C & C & A & C & C
\end{tabular}

B Haplotype distribution of DHCR7 mutations in 24 SLOS patients

\begin{tabular}{ll}
\hline Mutation & Haplotype $^{\text {a }}$ \\
\hline IVS8-1G>C & A (13); E (1 $1^{\text {b }}$ ) \\
R404C & B (1); D (1); F (3) \\
T93M & A (1); E (2 $)$ \\
W151X & C (2); F (2); G (3) \\
V326L & A (5) \\
R352W & A (4); I (1) \\
L99P & B (2) \\
E448K & A (1) \\
T289I & A (1) \\
G410S & A (1) \\
Y324Q & A (1) \\
A247V & B (1) \\
Q107H & H (1) \\
R443C & A (1) \\
\hline
\end{tabular}

${ }^{a}$ number of alleles in parenthesis. ${ }^{\mathrm{b}}$ one E allele carries both mutations: IVS8-1G $>C$ and T93M (see text).

mutation. One of the other T93M alleles was also characterised by this haplotype. Hence the double mutant likely arose by recombination. Together the haplotype data suggest that IVS8-1G >C and V329L are founder mutations.

\section{Discussion}

With the exception of two small studies, one from the Netherlands involving three patients ${ }^{17}$ and one Italian involving nine SLOS patients ${ }^{24}$ no data on the frequencies of SLOS-causing mutations in the DHCR7 gene in individual European populations are at present available. Frequency spectra of DHCR7 mutations on SLOS chromosomes are only known for American ${ }^{19}$ and a mixed American/European collection $^{18}$ of patients. Most, though not all of these patients, are of European ancestry, but for most the precise ancestry was unknown or not specified. In these studies six frequent DHCR7 mutations IVS8-1G >C, W151X, V326L, R352W, R404C and T93M) all with frequencies $>0.05$ of SLOS chromosomes were identified. In the present work we have analysed the spectra of DHRC mutations on SLOS chromosomes from three European countries representing east (Poland), central (Germany/Austria) and northwest (Great Britain) Europe. This analysis revealed extreme frequency gradients (Table5) from west to east and vice versa and together with data from Italy ${ }^{24}$ supports the notion that
Table 5 Frequencies of common mutations in the DHCR7 gene in three European populations

\begin{tabular}{|c|c|c|c|}
\hline Mutation & Poland & $\begin{array}{l}\text { Germany/ } \\
\text { Austria }\end{array}$ & Great Britain $^{a}$ \\
\hline IVS8-1G>C & 0.03 & 0.20 & $0.34(0.46)$ \\
\hline W151X & 0.33 & 0.18 & $0.02(0.04)$ \\
\hline V326L & 0.23 & 0.18 & 0 \\
\hline R352W & 0.13 & 0.07 & 0 \\
\hline R404C & 0 & 0.02 & 0.09 (0.07) \\
\hline T93M & 0 & 0 & $0.07(0.04)$ \\
\hline Total frequency & 0.72 & 0.65 & $0.52(0.61)$ \\
\hline
\end{tabular}

adata in parentheses consider only patient identified in Great Britain.

frequent SLOS-causing mutations have different geographic origins and histories. Multiple origins in Europe have been described for other disease-causing mutations, eg in phenylalanine hydroxylase (PHA) in patients with phenylketonuria. $^{27}$

As noted previously ${ }^{18}$ it is intriguing that two of the most common SLOS-causing mutations are null mutations. We do not know the origin or age of any of these and their exact population frequencies are at present unknown. We did not observe a single consanguineous marriage among the 59SLOS families which might indicate that DHCR7 mutations are more common than suggested by the reported incidence of SLOS. Only for the common IVS8-1G >C mutation limited population data are available. This mutation was observed once among 90 normal adult Caucasian Americans but not among Africans, Chinese, Japanese, and Finns. ${ }^{19}$ The latter is compatible with the virtual absence of the SLOS from Finland (H. Kääriänen, 1999, personal communication). The frequency distributions observed here in SLOS patients can be explained by different scenarios. One is that there is a high frequency of recurrent mutations at the DHCR7 locus which has created different random frequency spectra in European populations. However, this seems unlikely in view of the presence of frequency gradients and a very high relative frequency of some mutations (eg IVS8$1 G>C$ ). Further, none of the frequent null mutations (IVS8$1 G>C$ and $W 151 X)$ involve CpGs, whereas some other frequent mutationsie T93M, R352W, and R404C are at CpGs. Our limited haplotype analysis is consistent with this interpretation. All IVS8-1G >C alleles $(n=13)$ and all V326L alleles $(n=5)$ shared the same haplotype. Almost two-thirds of all DHCR7 mutations occurred on this haplotype. But we do not yet know the frequency of this common SLOS haplotype in the general population. Some of the mutations involving CpGs (R352W, T93M, and R404C) occurred on chromosomes with different DHCR7 haplotypes. Hence there is evidence also from haplotype analysis for both recurrent mutations and founder effects. This situation is still unclear for the W151X mutation. This mutation, despite the clear frequency gradient, occurred on three different haplotypes (Table4B). These differed only at single nucleotide positions and therefore may all be derived from the same 
ancestral haplotype. Since we do not yet know the frequencies and evolutionary relationships of DHCR7 haplotypes we can not yet distinguish between the possibility that W 151X is a recurrent mutation or is very old and has spread to different haplotypes. Since W151X is not due to a CpG and shows a very pronounced gradient, we favour the latter hypothesis.

The presence of opposite gradients from west to east and east to west of several DHCR7 mutations is not easily explained by the demic diffusion model of the peopling of Europe by neolithic farmers, which is believed to explain some of the gene frequency gradients in Europe (first principle component). ${ }^{28,29}$ Rather, a more complex situation seems to exist. Among the populations studied the frequency of the IVS8-1G >C mutation is highest in Britain. This mutation is obviously rare in South and East Europe. It was not observed among 18SLOS alleles from southern Italy ${ }^{24}$ and is rare in Poland (see Table1). Its frequency increased towards the northwest. This mutation may therefore characterise northern European populations. The high frequency and distribution of the IVS8-1G >C mutation could reflect migrations and/or replacement of ancient European populations carrying this mutation, as has been suggested for the $\Delta$ F508 mutation causing cystic fibrosis. ${ }^{30}$ In support of the notion that the IVS8-1G >C is old is our previous observation that two SLOS chromosomes carried two different mutations. Both double mutant alleles involved the IVS8IG >C site together with either R352Q (which is rare) or T93M which is frequent in Italy. Less likely, such double mutation alleles might have arisen by intragenic recombination. It is however intriguing that the parents of one of the patients (D111 in Witsch-Baumgartner ${ }^{18}$ ) with a double mutant allele (genotype IVS8-1G >C/T93M/IVS8-1G >C) had Irish/English ancestors on the maternal side and Italian ancestors on the paternal side. Haplotype analysis of this patient together with the haplotype distribution of IVS8IG >C and T93M mutations is indeed consistent with a recombination event (Table4B).

Notably the frequency gradient of the W151X mutation is in a direction opposite to the gradient of the IVS8-1G >C mutation. A similar gradient has been described for the R408W mutation in the PHA gene on haplotype2 chromosomes from patients with phenylketonuria. ${ }^{27}$ This gradient has been related to an east-west cline in polymorphic marker frequencies believed to reflect the so-called Kurgan expansion of pastoral nomads speaking Indo-European languages which occurred between 4300 and $3000 \mathrm{BC}$. There have been further extensive migrations in Europe over the last 2000 years however, and even quite recently, eg there was a large immigration from Poland to the coal-mining areas of the Ruhr district in Germany in the nineteenth century. Such migrations may well explain the frequency gradient of the 'Polish' W151X mutation. Hence the distribution pattern of DHCR7 mutations in Europe as it starts to emerge from this study might be a reflection of ancient and modern migra- tionsin Europe. In a recent study De Brasi et al ${ }^{24}$ reported that T93M is the most frequent DHCR7 mutation in Italian SLOS patients. Thus the pattern of SLOS mutations in Caucasians may be more complex than indicated by the present data. The R404C mutation which is the second most frequent in the British SLOS patients was previously identified in homozygous form in related families of French ancestry from a Louisiana parish in the USA and considered a French founder mutation. ${ }^{18}$ It will be interesting to see whether and in what frequency this mutation is present on French SLOS chromosomes and whether it spread from France to Britain with, for example, the Norman invasion. T93M is also among the three most frequent mutations in Great Britain but was absent in the German/Austrian and Polish samples. Whether this reflects ancient migrations (eg the Roman occupation of the southern parts of Great Britain) or still another cline needs to be clarified by analysis of further populations (such as France) in combination with haplotype analysis.

A more detailed analysis of the frequencies and hapl otypes of the common mutations in various European populations may not only shed more light on their origins and ages but also may give new insights into the genetic structure and migrations of European populations. It might also provide clues towards possible selective forces explaining the high frequency of DHCR7 mutations in Europeans and their absence in most non-Europeans.

Finally our results have also practical implication since they provide strategies for mutation detection in different European regions.

\section{Acknowledgements}

Wethank Drs P Bittigau, G Krüger. P M einecke, $\mathrm{H}$ Thiele, B Utermann, $\mathrm{U}$ Wendel, $\mathrm{M}$ and $\mathrm{N}$ Blandfort, $\mathrm{H}$ Zierler, and $\mathrm{H}$ Rehder for samples and for clinical data on their patients with SLOS. The skillful technical assistance of Ramona Berberich is gratefully acknowledged. BU Fitzky was supported by the Austrian Science Fund grant P 11636 to $\mathrm{H}$ Glossmann. This work was supported by the Austrian Science Fund grant P 12792-GEN to GU. MW -B and EC contributed equally to this work.

\section{References}

1 Smith D, Lemli L, Opitz J: A newly recognized syndrome of multiple congenital anomalies. J Pediatr 1964; 64: 210-217.

2 Opitz JM: RSH/SLO ('Smith-Lemli-Opitz') syndrome: Historical, genetic, and developmental considerations (review). Am J Med Genet 1994; 50: 344-346.

3 Kelley RI, Hennekam RCM: The Smith-Lemli-Opitz syndrome. J Med Genet 2000; 37: 321-335.

4 Bzduch V, Beholova D, Skoduva J: Incidence of Smith-Lemli-Opitz syndrome in Slovakia. Am J Med Genet 2000; 90: 260.

5 Opitz JM: RSH (so-called Smith-Lemli-Opitz) syndrome. Curr Opin Pediatr 1999; 4: 353-362.

6 Kelley RI: RSH/Smith-Lemli-Opitz Syndrome. Am J Hum Genet 1998; 63: 322-326.

7 Fitzky BU, Glossmann H, Utermann G, Moebius FF: Molecular genetics of the Smith-Lemli-Opitz syndrome and postsqualene sterol metabolism. Curr Opin Lipid 1999; 10: 123-131. 
8 Cunniff C, Kratz LE, Moser A, Natowicz MR, Kelley RI: Clinical and biochemical spectrum of patients with RSH/Smith-LemliOpitz Syndrome and abnormal cholesterol. Am J Med Genet 1997; 68: 263-269.

9 Kelley RI: A new face for an old syndrome. Am J Med Genet 1997; 65: 251-256.

10 Angle B, Tint GS, Yacoub OA, Clark AL: Atypical case of SmithLemli-Opitz syndrome: implications for diagnosis. Am J M ed Genet 1998; 80: 322-326.

11 Nowaczyk MJM, Whelan DT, Hill RE: Smith-Lemli-Opitz syndrome: Phenotypic extreme with minimal clinical findings. Am J Med Genet 1998; 78: 419-423.

12 Irons M, Elias ER, Salen G, Tint GS, Batta AK: Defective cholesterol biosynthesis in Smith-Lemli-Opitz Syndrome. Lancet 1993; 341: 1414.

13 Tint GS, Irons M, Elias ER et al: Defective cholesterol biosynthesis associated with the Smith-Lemli-Opitz Syndrome. N Engl J Med 1994; 330: 107-113.

14 Moebius FF, Fitzky BU, Lee JN, Paik Y-K, Glossmann H: Molecular cloning and expression of the human $\Delta 7$-sterol reductase. Proc Natl Acad Sci USA 1998; 95: 1899-1902.

15 Fitzky BU, Witsch-Baumgartner M, Erdel $M$ et al: Mutations in the $\Delta 7$-sterol reductase gene in patients with the Smith-Lemli-Opitz Syndrome. Proc Natl Acad Sci USA 1998; 95: 8181-8186.

16 Wassif CA, Maslen C, Kachilele-Linjewile S et al: Mutations in the human sterol $\Delta 7$-reductase gene at 11q12-13 cause Smith-LemliOpitz syndrome. Am J Hum Genet 1998; 63: 55-62.

17 Waterham HR, Wijburg FA, Hennekam RCM et al: Smith-LemliOpitz Syndrome is caused by mutations in the 7-dehydrocholesterol reductase gene. Am J Hum Genet 1998; 63: 329-338.

18 Witsch-Baumgartner M, Fitzky BU, Ogorelkova $M$ et al: Mutational spectrum in the $\Delta 7$-sterol reductase gene and genotypephenotype correlation in 84 patients with Smith-Lemli-Opitz Syndrome. Am J Hum Genet 2000; 66: 402-412.

19 Yu H, Tint GS, Salen G, Patel SB: Detection of a common mutation in the RSH or Smith-Lemli-Opitz syndrome by a PCR-RFLP assay: IVS8-G- $>$ C is found in over sixty percent of US propositi. Am J Med Genet 2000; 90: 347-350.
20 Waterham HR, Oostheim W, Romeijn GJ, Wanders RJA, Hennekam RC: Incidence and molecular mechanism of aberrant splicing owing to a G- >C splice acceptor site mutation causing SmithLemli-Opitz syndrome. J Med Genet 2000; 37: 387-389.

21 Löffler J, Trojovsky A, Casati B, Kroisel PM, Utermann G: Homozygosity for the W151X stop mutation in the $\Delta 7$-sterol reductase gene (DHCR7) associated with a lethal form of SmithLemli-Opitz syndrome - retrospective molecular diagnosis. Am J Med Genet 2000, (in press).

22 Kelley RI: Diagnosis of Smith-Lemli-Opitz Syndrome by gas chromatography/mass spectrometry of 7-dehydrocholesterol in plasma, amniotic fluid and cultured skin fibroblasts. Clin Chim Acta 1995; 236: 45-58.

23 Krajewska-Walasek M, Gradowska W, Ryzko J et al: Further delineation of the classical Smith-Lemli-Opitz syndrome phenotype at different patient ages: clinical and biochemical studies. Clin Dysmorph 1999; 8: 29-40.

24 DeBrasi D, Esposito T, Rossi M et al: Smith-Lemli-Opitz syndrome: evidence of T93M as a common mutation of delta7-sterol reductase in Italy and report of three novel mutations. Eur J Hum Genet 1999; 8: 937-940.

25 Neklason DW, Andrews KM, Kelley RI, Metherall JW: Biochemical variants of Smith-Lemli-Opitz syndrome. Am J Med Genet 1999; 85: 517-523.

26 Patrono C, Rizzo C, Tessa A et al: Novel 7-DHCR mutation in a child with Smith-Lemli-Opitz syndrome. Am J Med Genet 2000; 91: $138-40$.

27 Eisensmith RC, Okano $Y$, Dasovich $M$ et al: Multiple origins for phenylketonuria in Europe. Am J Hum Genet 1992; 51: 1355-1365.

28 Menozzi P, Piazza A, Cavalli-Sforza L: Synthetic maps of human gene frequencies in Europeans. Science 1978; 201: 786-792.

29 Cavalli-Sforza LL, Menozzi P, Piazza A: Demic expansions and human evolution. Science 1993; 259: 639-646.

30 Morral N, Bertranpetit J, Estivill $X$ et al: The origin of the major cystic fibrosis mutation (DeltaF508) in European populations. Nat Genet 1994; 7: 169-175. 